\title{
Closing in on the properties of antihydrogen
}

\author{
Michael Charlton ${ }^{\mathrm{a}}$ \\ Department of Physics, College of Science, Swansea University, Swansea SA2 8PP, UK
}

Received 13 July 2017 / Received in final form 17 August 2017

Published online 23 November 2017

(C) The Author(s) 2017. This article is published with open access at Springerlink.com

\begin{abstract}
Recent achievements leading, amongst other things, to the observation of the two-photon $1 \mathrm{~S}-2 \mathrm{~S}$ transition in antihydrogen are reviewed. We summarise some of the technical advances that were necessary to facilitate this, and how these addressed the requirements posed by the underlying physical processes. Aspects of the latter have been elucidated by simulations aimed at describing as closely as possible the experimental situations, and we describe some recent insights from that work. We will close with a look to the future.
\end{abstract}

\section{Introduction}

"We have performed the first laser-spectroscopic measurement on an atom of antimatter. This milestone has long been the most sought-after achievement in low-energy antimatter physics" [1]. Quite! In the author's case the wait (well worth it) was just over 30 years.

This monumental advance followed more than two decades of development and physics progress. Antihydrogen $(\overline{\mathrm{H}})$ was first produced via the controlled mixing of clouds of positrons $\left(e^{+}\right)$and antiprotons $(\bar{p})$ by the ATHENA collaboration in 2002 [2]. Though $\vec{H}$ trapping was a goal from the early days of the field [3,4], it was not achieved until 2010 [5]. It was then quickly discovered that the $\overline{\mathrm{H}}$ survived many minutes in the extreme, cryogenic high vacuum present in the ALPHA magnetic minimum neutral atom trap [6]. This important result showed that it was feasible to attempt experiments that required long interaction times with ground state $\bar{H}$ (such as the aforementioned laser study - the observation of the two-photon $1^{3} \mathrm{~S}-2^{3} \mathrm{~S}$ transition [1]).

With this underlying capability demonstrated, ALPHA has gone on to perform a series of ground-breaking measurements on $\overline{\mathrm{H}}$ including: observation of ground state hyperfine transitions [7], measurements of the charge neutrality of the anti-atom $[8,9]$ and the demonstration of a method of measuring its gravitational mass [10]. And as described in Section 5, there is promise of much more to follow.

Antihydrogen trapping apparatus [11] consists of a number of elements, with two of the most crucial being

\footnotetext{
* Contribution to the Topical Issue "Low energy positron and electron interactions", edited by James Sullivan, Ron White, Michael Bromley, Ilya Fabrikant, and David Cassidy.

${ }^{a}$ e-mail: m.charlton@swansea.ac.uk
}

the charged particle traps and the neutral atom trap. The former are typically Penning-Malmberg traps in which an array of cylindrical electrodes is suitably biased to effect a confining potential along the axis of a solenoid whose field, typically in the Tesla region, provides radial confinement for the $e^{+}$and $\bar{p}$. The multipolar (see Sect. 2) neutral atom trap is superimposed on the charged particle trap region. With its spatially increasing magnetic field both axially and radially, confinement can be effected upon $\overline{\mathrm{H}}$ with a suitable magnetic moment (i.e, the low-field seekers) and if they have a low enough kinetic energy $(0.54 \mathrm{~K}$ expressed in temperature terms for ALPHA [11]). It is in this environment, with a mixture of electric and (strong and spatially varying) magnetic fields that $\bar{H}$ is currently created, trapped and studied.

In most experiments the positron density $\left(n_{e}\right)$ and temperature $\left(T_{e}\right)$ conditions are such that $\overline{\mathrm{H}}$ formation is likely to be dominated by the three-body reaction,

$$
e^{+}+e^{+}+\bar{p} \rightarrow \overline{\mathrm{H}}+e^{+}
$$

As described elsewhere [12,13], this reaction has a strong dependence on $T_{e}$ (proportional to $T_{e}^{-9 / 2}$ in equilibrium), and the nature of the $e^{+}-e^{+}$energy exchange means that typical binding energies are in the region of $k_{\mathrm{B}} T_{e}$, with $k_{\mathrm{B}}$ being Boltzmann's constant. Such weakly bound states are strongly affected by ambient fields and collisions, and aspects of this will be described in Section 3.

The remainder of the article is organised as follows. The following section provides a summary of the experiment, whilst Section 3 gives details of simulations of reaction (1), and some of the pertinent results. Selected experimental achievements are discussed in Section 4, whilst we indulge in some speculation when closing with Section 5 . 


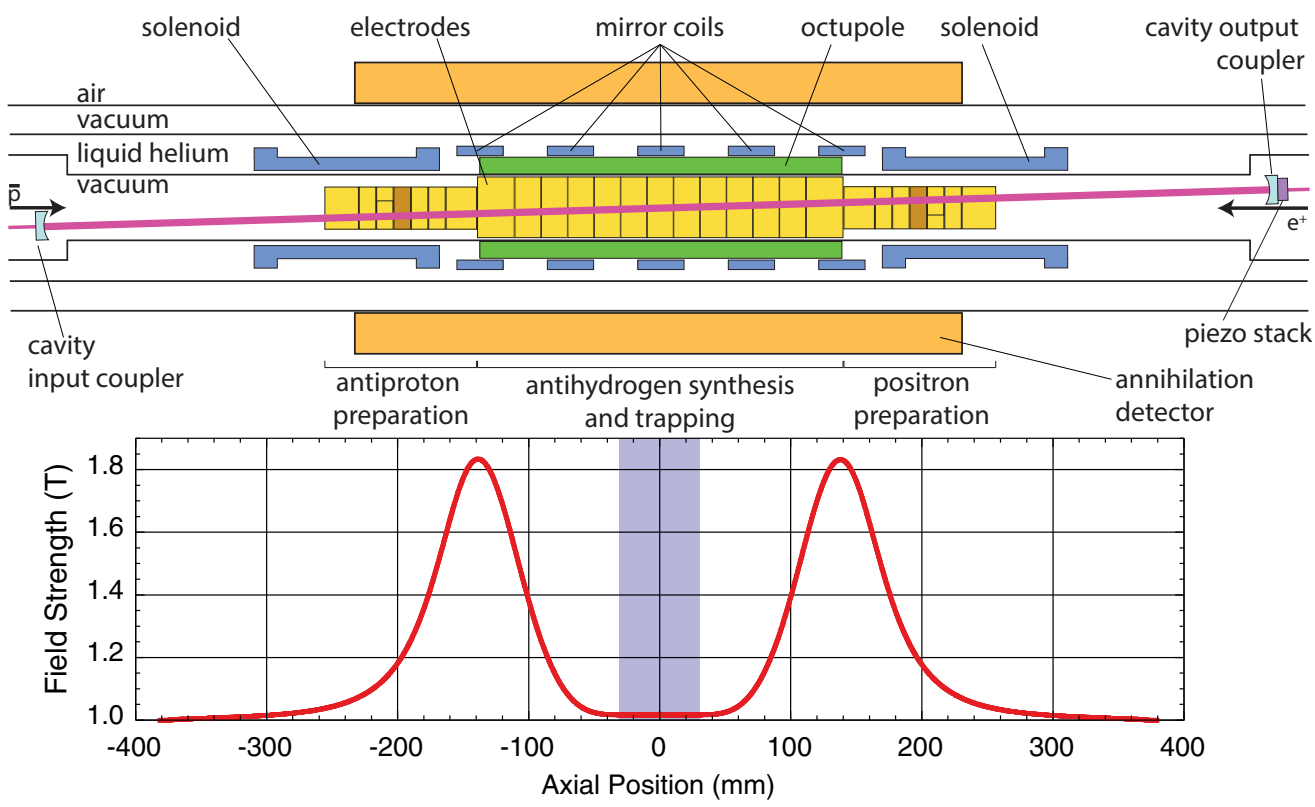

Fig. 1. Schematic illustration of the ALPHA2 apparatus used for the $1^{3} \mathrm{~S}-2^{3} \mathrm{~S}$ experiment [1]. The various Penning trap electrodes are shown, including those for the $\bar{p}$ and $e^{+}$preparation regions, which are themselves fed by the catching trap and buffer gas accumulator respectively (see text). The extra solenoids provide confining fields for the charged particles in these regions. The neutral atom trap is comprised of the set of mirror coils and the octupole. A schematic of the laser cavity is shown. The $243 \mathrm{~nm}$ light enters from the left side and crosses the central region of the trap at an angle of $2.3^{\circ}$. The annihilation detector is shown in block form (with its actual radial extent being larger than shown) and consists of a 3-layer silicon device, as explained further in the text.

\section{Experimental details}

A schematic illustration of the inner trapping region of the ALPHA experiment is shown in Figure 1. Here the cylindrical electrodes of the charged particle traps are shown with the superconducting neutral atom trap superimposed. The latter is comprised (in this version of the apparatus) of an octupolar coil system which provides radial confinement of the anti-atoms, provided they have low enough kinetic energies. Axial confinement is effected using a set of mirror coils, with the most basic configuration being with just those at either end energised to act as simple mirror coils. The depth of the magnetic trap for ground state $\overline{\mathrm{H}}$ is $\mu_{\mathrm{B}} \Delta B$, with $\mu_{\mathrm{B}}$ the Bohr magneton and $\Delta B$ the field change across the trap and amounts to around $0.7 \mathrm{~K}$ per Tesla. As mentioned in Section 1 the ALPHA trap is about $0.5 \mathrm{~K}$ deep [11], which poses great challenges in maximising the yield of trapped $\hat{H}$, given the comparison of this energy scale with the electrostatic self energy of the positron clouds used, which is of order eV.

Positrons and antiprotons are initially collected and manipulated in separate parts of ALPHA [11], not shown in Figure 1. The positron buffer gas accumulator is a device that can collect around $10^{8} e^{+}$in a few minutes [14] before transferring them [15] to the $\overline{\mathrm{H}}$ production and trapping region. ALPHA also now utilises a standalone catching-trap to capture and cool $\bar{p}$ s ejected from CERN's Antiproton Decelerator (AD) [16] using well-tried techniques (see $[12,13,17]$ for reviews).
To promote $\overline{\mathrm{H}}$ trapping, antiparticle clouds as cold as possible need to be prepared, and as close as possible to the axis of the traps. The latter is to avoid deleterious effects resulting from the radial variation of the octupolar field [18], and to minimise the effect of plasma rotation (caused by the $e^{+}$plasma self electric field and the axial applied magnetic field) on the $\overline{\mathrm{H}}$ kinetic energy $[19,20]$. As a result evaporative [21] and adiabatic [22] cooling methodologies have been developed by the different experiments, whilst compression techniques (see, e.g., [23,24]) have allowed the density and radial extent of the clouds to be tailored. This has been accompanied by refinements in $\bar{p}-e^{+}$mixing techniques $[1,25]$ which have facilitated marked improvements in $\overline{\mathrm{H}}$ trapping efficiencies, from about $5 \times 10^{-5}$ in the earliest work [5] to around $5 \times 10^{-4}$ in a recent study [26].

Another crucial feature of ALPHA (only shown in outline in Fig. 1) is a three-layer silicon detector which surrounds the mixing and trapping region. This instrument is able to efficiently reconstruct $\bar{p}$ annihilation vertices (annihilation involves the emission of several energetic pions), thereby locating the radial and axial position of the event. Furthermore, careful analysis of the recorded signals from the detector can be used isolate $\bar{p}$ and $\overline{\mathrm{H}}$ annihilations from events due cosmic rays [27]. The latter are counted at a rate of $10-11 \mathrm{~s}^{-1}$ and constitute a background which must be suppressed to allow investigations involving trapped $\overline{\mathrm{H}}$.

As described in Section 4, several experiments performed to date rely upon using effects to convert trapped, 
low magnetic field seeking states, into high-field seekers, which immediately leave the trap and annihilate. A detector so-called appearance measurement isolates these events over the sometimes several minutes of a run: something which puts stringent requirements on the event reconstruction to discriminate against cosmics (see e.g., [1]). A disappearance measurement involves emptying the trap in a short (typically around a second) time after the experiment has been performed and counting the $\overline{\mathrm{H}}$ annihilations and comparing this signal to runs in which the low-high field seeking transition was not excited. The narrow time window allows some of the event requirements to be relaxed, resulting in a higher overall efficiency, albeit at the expense of a lower discrimination against backgrounds.

Recent ALPHA experiments have seen the addition of a special cryogenic region which can allow microwave and laser ingress into the apparatus. The microwaves are fed into the apparatus using a purpose-built vacuum feedthrough, and guided down the axis of the charged particle traps to interact with the trapped $\overline{\mathrm{H}} \mathrm{s}$ as they pass through the minimum magnetic field at the trap centre. The frequencies of the hyperfine transitions involved are clearly dependent upon the magnitude of the field such that those corresponding to the trap centre are specially selected [7]. More recently, ALPHA has installed access for laser light, and in particular at $243 \mathrm{~nm}$, which has been used to excite the Doppler free two-photon $1^{3} \mathrm{~S}-2^{3} \mathrm{~S}$ transition [1]. This has entailed the development of a special cavity to build up power to ensure that an observable fraction of the $\overline{\mathrm{H}}$ undergoes the transition. More details can be found in [1] and in Section 4.

\section{Simulations}

In this section we briefly describe some of the key insights to have emerged from simulations of $\bar{H}$ formation via $e^{+}-\bar{p}$ mixing. Theoretical and computational studies of reaction (1) have, as reviewed by Robicheaux [28], been undertaken for some time. Here we will concentrate on the later work of Jonsell and co-workers [19,20,29] who have conducted extensive simulations for ranges of parameters (e.g., $n_{e}$ between $10^{13}$ and $10^{15} \mathrm{~m}^{-3}, T_{e}$ from 10 to $50 \mathrm{~K}$ and magnetic fields in the Tesla regime) of relevance to experiment.

The simulations are based upon the use of classical equations of motion, which is reasonable considering the distance scales involved: quantum effects will only be important for more tightly bound $\overline{\mathrm{H}}$ states than considered here. (Recall from Sect. 1 that reaction (1) produces loosely bound $\bar{H}$.) The axial magnetic field is included, as is the self electric field of the $e^{+}$plasma, which is radial $(r)$ in nature and has a strength proportional to $n_{e} r$. Radiative effects, including direct radiative $\overline{\mathrm{H}}$ formation $\left(\bar{p}+e^{+} \rightarrow \overline{\mathrm{H}}+h \nu\right)$, are not included as they are not expected to contribute greatly at the positron temperatures considered, nor at the early stages of $e^{+}-\bar{p}$ interaction when stable $\overline{\mathrm{H}}$ is formed.

Rather than present a detailed picture of the findings, we summarise some of the main results; more details can be found in the aforementioned works.
- The $\overline{\mathrm{H}}$ that is observed experimentally (i.e., that escapes the positron plasma and annihilates on the trap electrodes, or is held in the minimum- $B$ trap) is the result of repeated cycles of formation and break-up, with the latter being the reverse of reaction (1).

- Typically a $\bar{p}$ spends less than $1 \%$ of its time in the $e^{+}$plasma as $\overline{\mathrm{H}}$, since once it is formed it is quickly broken up.

- Antiprotons are transported radially by normal thermal diffusion AND when neutralised as $\overline{\mathrm{H}}$.

- When $\overline{\mathrm{H}}$ formation is important (i.e., at low $T_{e}$ ) the latter transport mechanism dominates and it is a form of ballistic diffusion.

- $\overline{\mathrm{H}}$ formed by the three-body reaction is typically not in a favoured magnetic moment state for capture in the minimum- $B$ trap [30], however $e^{+}$collisions help to randomise this and increase the trapped fraction [29].

\section{Selected experimental results}

To date, only the ALPHA collaboration have reported the results of experiments exploring the properties of the $\overline{\mathrm{H}}$ atom and we summarise some of that work here. The foundations for pursuing the spectroscopy of $\overline{\mathrm{H}}$ have been set by observations of ground state hyperfine [7] and the two-photon [1] $1^{3} \mathrm{~S}-2^{3} \mathrm{~S}$ transitions.

For the hyperfine measurement, trapped $\overline{\mathrm{H}}$ atoms were exposed to microwaves at frequencies around $29 \mathrm{GHz}$ (corresponding to the positron spin flip transition frequency in the $1 \mathrm{~T}$ magnetic field at the minimum of the neutral atom trap) to drive the transitions from the low-field to highfield seeking, untrapped, states - usually termed the $|d\rangle$ to $|a\rangle$ and $|c\rangle$ to $|b\rangle$ transitions. The two transitions were addressed independently using $15 \mathrm{MHz}$ wide scans across the respective expected onset frequency. These were interleaved with off-resonance measurements via a $100 \mathrm{MHz}$ detuning followed by a further on-resonance measurement in which the magnetic field was changed to compensate for the frequency shift. The aforementioned appearance and disappearance signals were analysed. The former are shown in Figure 2 where a clear excess of counts is visible in the on-resonance case, compared to the data offresonance and with no microwaves. For the disappearance mode the number of $\overline{\mathrm{H}}$ s remaining in the trap expressed as a rate (number per attempt) in the three cases were $0.02 \pm 0.01$ (on-resonance), $0.21 \pm 0.04$ (off-resonance) and $0.40 \pm 0.06$ (no microwaves), again providing unambiguous evidence that the low- to high-field seeking transitions had been excited. (The reason the off-resonance result is lower than that for no microwaves is connected to the magnetic field variation in the minimum- $B$ trap: see [7] for more details.)

The two-photon $1{ }^{3} \mathrm{~S}-2^{3} \mathrm{~S}$ experiment was conducted in the new apparatus called ALPHA-2 which, as shown in Figure 1 contains a versatile magnetic minimum trap and laser access with a build-up cavity for the required light at $243 \mathrm{~nm}$. The laser system produces about $1 \mathrm{~W}$ of power focussed onto the centre of the trap to induce transitions 


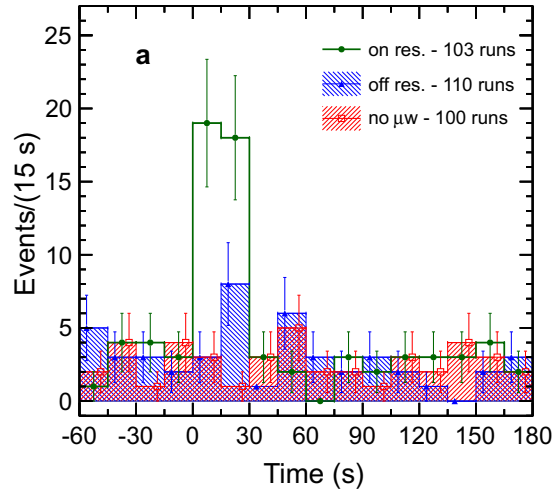

Fig. 2. Appearance data for the $\overline{\mathrm{H}}$ microwave experiment versus exposure time, with the microwaves applied at $t=0$. The two possible quantum jumps were each subject to $15 \mathrm{~s}$ bursts of microwaves, repeated over the $180 \mathrm{~s}$ duration of the trial. A trial consisted of forming antihydrogen, and then waiting an appropriate time (typically a second or so) to ensure that the excited states produced via reaction (1) had decayed to the ground state before commencing irradiation. There is a clear excess of counts in the two first time bins, indicating that the low- to high-field seeking transitions have been excited. With hindsight, the microwave power used meant that almost all the $\overline{\mathrm{H}}$ atoms were expelled from the trap during the first $15 \mathrm{~s}$ burst. The on-resonance (green), off-resonance (blue hatched) and no microwaves (red hatched) each consisted of around 100 trials.

as close as possible to the minimum magnetic field. As mentioned elsewhere, the low-field seeking states (termed $1 S_{c}$ and $1 S_{d}$ ) survive in the trap and may be excited to corresponding states in the $2 \mathrm{~S}$ manifold. The frequencies $f_{\mathrm{c}-\mathrm{c}}$ and $f_{\mathrm{d}-\mathrm{d}}$ are slightly different due to the differences in hyperfine splitting between the ground and the excited states.

Following excitation to the $2 \mathrm{~S}$ state, the $\overline{\mathrm{H}}$ can be lost from the trap in two ways. First, motional electric fields can mix the $2 \mathrm{~S}$ and $2 \mathrm{P}$ states, allowing the $\overline{\mathrm{H}}$ to decay back to an untrapped ground state via the emission of a single photon. Secondly, an additional $243 \mathrm{~nm}$ photon can photoionise the excited $\overline{\mathrm{H}}$, with the resulting $\bar{p}$ lost radially from the trap [1]. Simulations showed the second mechanism to be dominant and that for the $300 \mathrm{~s}$ in which each of the transitions was excited about $50 \%$ of the $\overline{\mathrm{H}}$ should be lost, in accord with the observed value of $58 \pm 6 \%$. The experiment was run on-resonance, offresonance whereby the laser was detuned by $200 \mathrm{kHz}$ from the expected transition frequency (or more properly, half of it) and for no laser present during the combined $600 \mathrm{~s}$ hold time of the trapped $\overline{\mathrm{H}}$. Both appearance and disappearance mode data were taken (and were in accord) with the latter summarised as off-resonance $159 \pm 13$ events, no laser $142 \pm 12$ events and on-resonance with $67 \pm 8$ events. Thus the on- and off-resonance signal differ by $92 \pm 15$ with the transition frequencies (at twice those of the laser) found to be $f_{\mathrm{d}-\mathrm{d}}=2466061103064 \mathrm{kHz}$ and $f_{\mathrm{c}-\mathrm{c}}=2466061707104 \mathrm{kHz}$ each with a $400 \mathrm{kHz}$ (i.e., twice the laser shift) resolution. Both are in accord with expectations for transitions for hydrogen in the magnetic field of ALPHA's trap and provide, even in this first demonstration, a measurement at a few parts in $10^{10}$.
ALPHA has also undertaken investigations of the charge neutrality of $\overline{\mathrm{H}}[8,9]$ by applying electric fields to the anti-atom in an effort to expel it from the shallow minimum- $B$ trap. In the later of the two studies a stochastic acceleration technique was used to find that the putative charge was less 0.71 parts per billion in units of $e$. When assuming charge superposition and the best available determination of the $\bar{p}$ charge with respect to the proton of equality to within $5 \times 10^{-10}$, this could be used to set similar limits on the electron/positron charge ratio [31].

\section{Discussion and prospects}

The success of the antimatter programme at CERN has led the laboratory to invest in a significant upgrade to provide lower energy (100 keV rather than the AD output of $5.3 \mathrm{MeV}) \bar{p}$ s for experiments: the ELENA storage ring [32]. This will impact in two main ways. First, the lower kinetic energy will enhance $\bar{p}$ capture and cooling efficiencies, possibly by up to two orders of magnitude. Second, the delivery of $\bar{p}$ s to the experiments will be accomplished using electrostatic beamlines (rather than the magnetic transfer lines at present) which will be able to be switched more-or-less at will. Thus, the current system of block $\bar{p}$ allocation on an 8-h shift basis will be replaced by something akin to $\bar{p}$ s-on request, thereby increasing availability for all.

The near future will surely include $\overline{\mathrm{H}}$ spectroscopy whereby the on- and off-resonance studies carried out hitherto are replaced by scans across spectral lines, with the ground state hyperfine and $1^{3} \mathrm{~S}-2^{3} \mathrm{~S}$ transitions being the obvious first targets. The latter is known in hydrogen to around a few parts in $10^{15}$ [33] and thus holds the promise of a direct, very high precision matter-antimatter symmetry test. It is hoped that the laser frequency precisions mentioned in the previous section will soon be translated into direct $\overline{\mathrm{H}}$-hydrogen comparisons at a similar level.

ALPHA has also begun to make the first inroads into $\overline{\mathrm{H}}$ hyperfine spectroscopy [34] (so far to a precision of around a part in three thousand) and the ASACUSA collaboration has been working towards $\overline{\mathrm{H}}$ beam formation $[35,36]$ to conduct such measurements in a field-free environment. A recent test experiment on hydrogen has achieved a precision of a few parts in $10^{9}$ [37]. Given that excited states of $\overline{\mathrm{H}}$ are now available, it is also not out of the question to consider spectroscopy involving these levels, including a determination of the Lamb Shift.

The deployment of Lyman- $\alpha$ lasers with trapped $\overline{\mathrm{H}}$ is a short-term goal of both ALPHA and ATRAP. The aim here is not spectroscopy as such, but laser cooling. A numerical study [38] using realistic assumptions about laser parameters at the challenging Lyman- $\alpha$ wavelength, and incorporating features from ALPHA's minimum- $B$ trap, showed that $\overline{\mathrm{H}}$ may be cooled to around $20 \mathrm{mK}$. This would have obvious benefits for spectroscopy and studies aimed at probing the gravitational interaction of antimatter using what might be termed ballistic methods [10]. In this respect ALPHA is constructing a new vertical apparatus, ALPHA-g, which will complement ongoing efforts 
to measure $g$ for $\overline{\mathrm{H}}$ by the AEgIS [39] and GBAR [40] collaborations. It is expected that initial measurements will reveal the sign of antimatter gravity, with refinements and new techniques bringing further accuracy on $g$ for $\overline{\mathrm{H}}$. There has even been a suggestion that a measurement of this parameter might be possible at the $10^{-6}$ level [41] using an anti-atom fountain.

It is likely that the positronium (Ps) route to $\overline{\mathrm{H}}$ $\left(\bar{p}+\mathrm{Ps} \rightarrow \overline{\mathrm{H}}+e^{-}\right)$, which was demonstrated some time ago by Storry and co-workers [42] using a double charge exchange scheme [43], will be focus of future activity $[39,40,44]$. This is especially so given the recent experimental advances in producing excited states of Ps (see, e.g., [45-48]). Production of $\bar{H}$ in selected states is possible, by picking out the appropriate Ps state [49], and guidance on possible production rates using some excited states of Ps is now feasible with the availability of reliable theory $[50,51]$.

It is also possible, as first envisaged some time ago [52], and as revived by GBAR (e.g., [40]), to use the Ps charge exchange reaction to produce an antihydrogen beam from variable energy $\bar{p}$ projectiles. Such a beam could be used to investigate $\overline{\mathrm{H}}$ scattering, and also to produce the antihydrogen positive ion, $\overline{\mathrm{H}}^{+}$, (via $\overline{\mathrm{H}}-\mathrm{Ps}$ collisions) as envisaged by GBAR. This ion is likely to be of fundamental interest in its own right, and the Ps route to production appears to be the most efficient way, since the rate of direct radiative capture of a positron by a trapped $\overline{\mathrm{H}}$ atom is low [53]. A $\overline{\mathrm{H}}$ beam may also find application in further searches for a possible electric charge of the anti-atom. Finally, it may be feasible to use pulsed travelling optical lattices (as suggested for the formation of Ps beams [54]) to eject trapped $\overline{\mathrm{H}}$ in a beam whose properties are defined by those of the lasers used to generate the lattice.

A variant on the $\bar{p}$-Ps reaction for the production of $\overline{\mathrm{H}}$ is that discussed recently by Bertsche et al. [55], namely $\mathrm{X}^{+}+\mathrm{Ps} \rightarrow \mathrm{X}+e^{+}$to produce cold atomic species from cold trapped ions. Here a so-called hybrid ion-atom chip trap system was envisaged to produce and perhaps store the atoms. One can also envisage a cryogenic version of such a miniaturised apparatus (or some other scaled down trap system) for $\overline{\mathrm{H}}$ production and trapping, particularly if laser cooled negative ions can be used to sympathetically cool $\bar{p}$ s into the $\mathrm{mK}$ regime [56-58]. Much colder antihydrogen confined in a miniature trap would have undoubted benefits for spectroscopy.

There has been tremendous progress in the 15 years since cold antihydrogen was first produced. Fresh horizons are sure to open up with the arrival of CERN's new $\bar{p}$ capabilities, and we anticipate a very bright future for our field as we embark upon a journey of physics discovery.

I am grateful to the EPSRC (UK) and The Royal Society for supporting my work on antimatter. I thank all my colleagues in the ALPHA and ATHENA collaborations, without whom this adventure would not be the wonderful ongoing enterprise that it is. I am particularly grateful for recent collaborations with: C.J. Baker, W.A. Bertsche, A. Bianconi, I. Bray, E. Butler, A. Deller, D. Edwards, S. Eriksson, I.I. Fabrikant, A. Gutierrez, C.A. Isaac, S. Jonsell, A.S. Kadyrov, N. Madsen, T. Mortensen,
E. Lodi Rizzini, J.C. Straton, L. Venturelli and D.P. van der Werf. Finally, thanks Jeff. You made our 1S-2S dream happen.

Open Access This is an open access article distributed under the terms of the Creative Commons Attribution License (http://creativecommons.org/licenses/by/4.0), which permits unrestricted use, distribution, and reproduction in any medium, provided the original work is properly cited.

\section{References}

1. M. Ahmadi et al. (ALPHA collaboration), Nature 541, 506 (2017)

2. M. Amoretti et al. (ATHENA collaboration), Nature 419, $456(2002)$

3. G. Gabrielse, Hyperfine Interact. 44, 349 (1988)

4. M. Charlton, in Positron annihilation, edited by L. Dorikens Vanpraet, M. Dorikens, D. Segers (World Scientific, Singapore, 1988), pp. 181-190

5. G.B. Andresen et al. (ALPHA collaboration), Nature 468, 673 (2010)

6. G.B. Andresen et al. (ALPHA collaboration), Nat. Phys. 7, $558(2011)$

7. C. Amole et al. (ALPHA collaboration), Nature 483, 439 (2012)

8. C. Amole et al. (ALPHA collaboration), Nat. Commun. 5, 3955 (2014)

9. M. Ahmadi et al. (ALPHA collaboration), Nature 529, 373 (2016)

10. C. Amole et al. (ALPHA collaboration), Nat. Commun. 4, $1875(2013)$

11. C. Amole et al. (ALPHA collaboration), Nucl. Instrum. Methods Phys. Res. A 735, 319 (2014)

12. M.H. Holzscheiter, M. Charlton, M.M. Nieto, Phys. Rep. 402, 1 (2004)

13. G. Gabrielse, Adv. At. Mol. Opt. Phys. 50, 155 (2005)

14. C.M. Surko, R.G. Greaves, M. Charlton, Hyperfine Interact. 109, 181 (1997)

15. L.V. Jørgensen et al. (ATHENA collaboration), Phys. Rev. Lett. 95, 025002 (2005)

16. T. Eriksson, Hyperfine Interact. 194, 123 (2009)

17. W.A. Bertsche, E. Butler, M. Charlton, N. Madsen, J. Phys. B: At. Mol. Opt. Phys. 49, 064006 (2016)

18. J. Fajans, W. Bertsche, K. Burke, S.F. Chapman, D.P. van der Werf, Phys. Rev. Lett. 95, 155001 (2005)

19. S. Jonsell, M. Charlton, D.P. van der Werf, J. Phys. B: At. Mol. Opt. Phys. 49, 134004 (2016)

20. S. Jonsell, D.P. van der Werf, M. Charlton, F. Robicheaux, J. Phys. B: At. Mol. Opt. Phys. 42, 215002 (2009)

21. G.B. Andresen et al. (ALPHA collaboration), Phys. Rev. Lett. 105, 013003 (2010)

22. G. Gabrielse et al. (ATRAP collaboration), Phys. Rev. Lett. 106, 073002 (2011)

23. G.B. Andresen et al. (ALPHA collaboration), Phys. Rev. Lett. 100, 203401 (2008)

24. N. Kuroda et al., Phys. Rev. Lett. 100, 203402 (2008)

25. G.B. Andresen et al. (ALPHA collaboration), Phys. Rev. Lett. 106, 025002 (2011)

26. M. Ahmadi et al. (ALPHA collaboration), Nat. Commun. 8, $681(2017)$

27. G.B. Andresen et al. (ALPHA collaboration), Nucl. Instrum. Methods Phys. Res. A 648, 73 (2012) 
28. F. Robicheaux, J. Phys. B: At. Mol. Opt. Phys. 41, 192001 (2008)

29. S. Jonsell, M. Charlton, D.P. van der Werf, in preparation (2017)

30. F. Robicheaux, Phys. Rev. A 73, 033401 (2006)

31. M. Hori et al., Science 354, 610 (2016)

32. S. Maury et al., Hyperfine Interact. 229, 105 (2014)

33. C.G. Parthey et al., Phys. Rev. Lett. 107, 203001 (2011)

34. M. Ahmadi et al. (ALPHA collaboration), Nature 548, 66 (2017)

35. Y. Enomoto et al., Phys. Rev. Lett. 105, 243001 (2010)

36. N. Kuroda et al., Nat. Commun. 5, 3089 (2014)

37. M. Diermaier et al., Nat. Commun. 8, 15749 (2016)

38. P.H. Donnan, M.C. Fujiwara, F. Robicheaux, J. Phys. B: At. Mol. Opt. Phys. 46, 025302 (2013)

39. D. Krasnický et al. (AEgIS collaboration), Int. J. Mod. Phys.: Conf. Ser. 30, 1460262 (2014)

40. D.P. van der Werf, Int. J. Mod. Phys.: Conf. Ser. 30, 1460263 (2014)

41. M. Baquero-Ruiz et al., New J. Phys. 16, 083013 (2016)

42. C.H. Storry et al., Phys. Rev. Lett. 93, 263401 (2004)

43. E.A. Hessels, D.M. Homan, M.J. Cavagnero, Phys. Rev. A 57, 1668 (1998)
44. R. McConnell, et al. (ATRAP collaboration), J. Phys. B: At. Mol. Opt. Phys. 49, 064002 (2016)

45. D.B. Cassidy, T.H. Hisakado, H.W.K. Tom, A.P. Mills Jr, Phys. Rev. Lett. 108, 043401 (2012)

46. T.E. Wall et al., Phys. Rev. Lett. 114, 173001 (2015)

47. A. Deller et al., J. Phys. B: At. Mol. Opt. Phys. 48, 175001 (2015)

48. S. Aghion et al. (AEgIS collaboration), Phys. Rev. A 94, 012507 (2016)

49. M. Charlton, Phys. Lett. A 143, 143 (1990)

50. A.S. Kadyrov et al., Phys. Rev. Lett. 114, 183201 (2015)

51. C.M. Rawlins et al., Phys. Rev. A 93, 012709 (2016)

52. J.W. Humberston, M. Charlton, F.M. Jacobsen, B.I. Deutch, J. Phys. B: At. Mol. Phys. 20, L25 (1987)

53. C.M. Keating, M. Charlton, J.C. Straton, J. Phys. B: At. Mol. Opt. Phys. 47, 225202 (2014)

54. P.F. Barker, M. Charlton, New J. Phys. 14, 045005 (2012)

55. W.A. Bertsche, M. Charlton, S. Eriksson, New J. Phys. 19, $053020(2017)$

56. A. Kellerbauer, J. Walz, New J. Phys. 8, 45 (2006)

57. A. Kellerbauer, A. Fischer, U. Warring, Phys. Rev. A 89, 043430 (2014)

58. E. Jordan, G. Cerchiara, S. Fritzsche, A. Kellerbauer, Phys. Rev. Lett. 115, 113001 (2015) 